\title{
WIP: Entrepreneurially Minded Learning in a Physiological Signals Analysis Lab
}

Jennifer Bailey, Rochester Institute of Technology (COE)

Dr. Jennifer Bailey is a Senior Lecturer of Biomedical Engineering at Rochester Institute of Technology, where she has taught since January of 2014. She previously taught at the University of Illinois and the University of Southern Indiana after graduating from Purdue University. Bailey's passion is lab course development and improving student learning through enhancing lab and other hands-on experiences.

Dr. Michael Scott Richards 


\section{Entrepreneurially Minded Learning in a Physiological Signals Analysis Lab: Work in Progress}

\section{Introduction}

Lab courses are often designed with a specific objective that may or may not have a connection to the real-world problems. In addition, labs often offer a rigid, previously tested protocol, giving little to no room for flexibility by the students. These lab exercises do not allow for curiosity or creativity by the students and do not challenge their ability to reach beyond what is directly in front of them [1], [2]. The goal of this work is to change that pattern for application-oriented lab activities in the junior level Quantitative Physiological Signal Analysis Lab for Biomedical Engineering students by providing an opportunity for curiosity and creativity.

A few lab exercises, focused on application of signal processing concepts, were reformatted and translated closer to a problem-based learning (PBL) format. Entrepreneurially minded learning (EML) outcomes were added by providing real-world context with little explicit procedural direction [3]-[6]. Specific outcomes were provided to the students, see Table 1, while more outcomes were designed into the experience but not publicized to the students. The latter outcomes were as follows:

- Test different approaches to gather data (curiosity and creativity)

- Gather data to support or refute an idea (curiosity)

- Take ownership of, and express interest in a topic (curiosity)

- Recognize the personal impact of a design (creating value)

- Identify an unmet need (creating value)

- Present conclusions and recommendations with supporting evidence (communication)

\section{Methods}

Students take a one-credit Quantitative Physiological Signal Analysis Lab in their sixth semester. The course builds on concepts learned in their Biomedical Signal Analysis course and applies them to concepts learned in their Systems Physiology course from the previous semester. The initial module focuses on filter design and circuits. By week seven, students are collecting and analyzing physiological signals. Weeks 11, 12, and 13 were focused on electrooculography (EOG), electroencephalography (EEG), and electromyography (EMG), respectively. These lab exercises were modified closer to a PBL format [7] and EML learning outcomes were added. The cohort in the fall of 2019 consisted of three 3-hr sections with enrollments of 8, 21, and 19 students. Teams consisted of two students when possible or an occasional team of three.

A week before the lab, a handout was provided to the students with a question, the scenario being addressed by the question, the list of learning outcomes for the lab, the prelab expectations, materials with a sentence of methods explaining that the students need to develop the procedure, and a reminder of the two assessments required post-lab. Each of the three weeks required the students to complete a prelab before performing the lab activity and submit team notebooks and summaries. The purpose of the prelabs were to have the students learn about the origin of the signal and create an experimental plan for its collection and analysis, as none was provided by the instructors. The purpose of the notebooks was to document the students' method of collection 
and analysis of the physiologic signals. The purpose of the summaries was to address the posed question with their rationale.

Table 1. Relationship of physiological signal, the question posed to the students, the analysis that the instructors anticipated the students to perform, and the clearly stated learning outcomes provided to the students.

\begin{tabular}{|c|c|c|c|}
\hline Signal & Question & Anticipated Analysis & Learning Outcomes \\
\hline EOG & $\begin{array}{l}\text { Can EOG be used } \\
\text { during video game } \\
\text { testing to understand } \\
\text { how the user } \\
\text { interacts with the } \\
\text { game, specifically } \\
\text { what grabs their } \\
\text { focus? }\end{array}$ & $\begin{array}{l}\text { Show that direction and } \\
\text { the degree to which the } \\
\text { eye moves can be } \\
\text { determined from the } \\
\text { amplitude of the signal. } \\
\text { Demonstrate analysis. }\end{array}$ & $\begin{array}{l}\text { Collect EOG signals. } \\
\text { Use EOG signals to calculate } \\
\text { direction of view. } \\
\text { Evaluate feasibility of using } \\
\text { EOG for measuring visual } \\
\text { location. } \\
\text { Efficiently communicate results } \\
\text { including explanation. }\end{array}$ \\
\hline EEG & $\begin{array}{l}\text { Can EEG be used to } \\
\text { monitor semi-truck } \\
\text { drivers and } \\
\text { determine if they are } \\
\text { paying attention or } \\
\text { falling asleep while } \\
\text { driving? }\end{array}$ & $\begin{array}{l}\text { Alpha waves (8-15 Hz) } \\
\text { change amplitude with } \\
\text { opening/closing of eyes } \\
\text { and alertness. Beta } \\
\text { waves (16-31 Hz) change } \\
\text { amplitude with active } \\
\text { thinking. } \\
\text { Collect and perform a t- } \\
\text { test to compare. }\end{array}$ & $\begin{array}{l}\text { Collect EEG signals. } \\
\text { Analyze the frequency domain } \\
\text { of an EEG signal. } \\
\text { Evaluate feasibility of EEG for } \\
\text { determining attention or } \\
\text { tiredness. } \\
\text { Efficiently communicate results } \\
\text { including explanation. }\end{array}$ \\
\hline EMG & $\begin{array}{l}\text { Can EMG be used to } \\
\text { monitor fatigue in } \\
\text { assembly line } \\
\text { workers to better } \\
\text { schedule breaks or } \\
\text { job rotations? }\end{array}$ & $\begin{array}{l}\text { Verify that frequency } \\
\text { spectrum changes with } \\
\text { fatigue and then measure } \\
\text { that change. Demonstrate } \\
\text { it is predictable. }\end{array}$ & $\begin{array}{l}\text { Collect EMG signals. } \\
\text { Analyze the frequency domain } \\
\text { of an EMG signal. } \\
\text { Evaluate feasibility of EMG for } \\
\text { identifying and monitoring } \\
\text { fatigue. } \\
\text { Efficiently communicate results } \\
\text { including explanation. }\end{array}$ \\
\hline
\end{tabular}

Table 1 shows the summary of each lab. The full context of the scenarios was:

- EOG: A video game developer is creating a computer game and wants to ensure that certain characteristics in the game catch the user's attention. The developer is considering using EOG to understand what the user focuses on. Is this a feasible method?

- EEG: A transportation company wants to help its drivers be safe by anticipating when they are getting tired and should take a break and are wondering if EEG can help. A typical EEG cap is too much to ask a driver to wear but a few electrodes in a ball cap would be a possible solution. The company is asking for statistically significant results (ttest or ANOVA), not just preliminary data to make a decision.

- EMG: The company that manufactures our PowerLabs, AD Instruments, wants to prevent injuries to their assembly line workers by monitoring their level of fatigue. This will help them efficiently give their workers breaks and increase productivity by rotating 
their task on the line appropriately. Can EMG predict or indicate fatigue and help AD Instruments?

At the finish of the course, the students delivered a final oral presentation. The team chose one of these three lab exercises and presented their approach to answering the question. The presentation included their methods for signal collection, their results, and their conclusions regarding their ability to address the posed problem. Lastly, they were asked to identify and present a potential further application, other than the one given in the lab, for using the measured signal to solve a real-world problem.

\section{Results and Discussion}

The first and last outcomes provided to the students for each signal were fairly well achieved. Every team collected each signal as assessed by observation and notebooks and each team finished with an acceptably communicated summary even if analysis performed was incorrect. Occasionally more quantitative data or better technical presentation of the information was preferred. Overall, the students showed some creativity in their procedures and excellent curiosity and value creating opportunities for other problems that could be addressed.

To help students connect with question and reduce cognitive load on understanding the basic question being asked, scenarios were chosen that students could relate to on some level.

EOG: All engineering students use computers significantly and most students have played games on a computer.

EEG: While none have likely driven a large truck, many can relate to feeling of being fatigued when driving and all can relate to fatigue affecting their functioning on some task.

EMG: Most students probably have not seen an assembly line in-person so an assumption was that they could imagine a similar situation.

During the lab activities, an observation was that experimental procedures were more creative and accounted for more human factors as the teams conversed with more non-team members. These conversations appeared to help them gain additional perspectives and enhance their curiosity. Sometimes an instructor would mention an idea to one team who might later mention it to others or as teams would observe other teams collecting data in a different way, they would compare ideas. For example, some teams considered eye movement velocity when investigating EOG after discussing how people play video games.

Examples of creating value were displayed in the proposed further applications during their final presentations. One team suggested how EOG could help advance mental health treatment by identifying hallucinations in persons with schizophrenia. Another team suggested using EEG in human factors experiments to monitor cognitive overload when testing feasibility of a new device.

The primary concern from this experience is whether the students' knowledge of the engineering concepts was negatively impacted by this reformatting. The signal was not always collected correctly or in such a way that it could be analyzed to address the question leading to proper evaluation of feasibility. For example, conclusions from EOG data is dependent on how the 
signal was studied. Was eye movement velocity considered? Was the signal always relative to movement from a point of origin or was movement from many points considered? Were other human factors considered such as blinking or eye rubbing? If these factors were not considered during the lab time, the data cannot be extrapolated to answer these after lab when writing up the summary. For EEG, an occasional team would not collect data correctly or performed improper analysis which did not allow for a t-test to be performed appropriately. The assumption made by the instructors was that students knew how to perform an appropriate t-test but only provided clear guidance on how to include this in the experimental design to teams who asked.

Areas for Improvement:

1) Consider one lab for collecting basic signal data according to a more structured protocol followed by a second lab for collecting data to determine feasibility for an application. This would allow students to better understand the limitations of the signal, equipment, and their designed protocol.

For EOG and EEG signal measurement labs, students had no prior exposure to the experimental protocol. Thus, troubleshooting signal collection and the limitations with that process took more time than expected and left the students little time to consider any adjustments to their drafted procedure. Introducing these signal measurements earlier in the semester or introducing proper data collection techniques into the labs will be considered.

2) Give better details to the scenario and ask more probing questions to encourage more creative thinking.

For example, when students focused on the application of video game design, they limited their testing to computer screens and videos with one static object. Until further discussion with instructors, many did not consider moving objects or the idea of multiple objects distracting from the primary object. For the EMG exercise, students primarily tested the bicep muscle, which they had prior experience collecting a signal. This narrowed their ability to consider other muscles or muscle groups to test.

3) Raise expectations for feasibility evaluation specifically as it relates to how the technique would be implemented and impact the subject.

The EEG exercise specifically mentions the limitation of using a ball cap. However, other than addressing this instructor-imposed limitation, very few comments were made about personal limitations of the equipment on the subjects. For example, only a few EOG experimental summaries considered implementation concerns such as screen size and location of subject. In addition, only a few (out of 25) EOG conclusions considered normal human behavior that may corrupt their data such as blinking and squinting.

Overall, including scenarios in this PBL format is a recommended path for classes similar to this. Students are exposed to applications of the material instead of collecting and analyzing signals for the sole purpose of a class assignment. Students are given flexibility in their experimental 
designs which allows for creativity and curiosity. By proposing an additional application, students also appreciate how to create value.

\section{References}

[1] T. J. Kriewall and K. Mekemson, "Instilling the Entrepreneurial Mindset Into Engineering". The Journal of Engineering Entrepreneurship, vol. 1(1), pp. 5-19, 2010.

[2] D. Jamison, "Framework for Integrating Entrepreneurially Minded Learning in Upper Level Courses,” ASEE National Conference, Columbus OH, 2017.

[3] K. Moustaghfir and N.T. Sirca, "Entrepreneurial learning in higher education: introduction to the thematic issue,” International Journal of Euro-Mediterranean Studies, vol. 3.1, pp. 3-26, 2010.

[4] M. Lackeus, "Entrepreneurship in education - What, why, when, how, Entrepreneurship360 Background paper”, OECD, 2015.

[5] N. Duval-Couetil, T. Reed-Rhoads, and S. Haghighi, "Engineering students and entrepreneurship education: Involvement, attitudes and outcomes". International Journal of Engineering Education, vol. 28(2), pp. 425-435, 2012

[6] E. M. Eisenstein, 2010. "Engineering and entrepreneurship: Creating lasting value from engineering". IEEE Transforming Engineering Education: Creating Interdisciplinary Skills for Complex Global Environments, Dublin Ireland, 2010.

[7] R. M. Carey and R. D. Shonat, “Assessment of Self-Paced Laboratory Modules in Biomedical Engineering," ASEE/IEEE Frontiers in Education Conference, Indianapolis IN, 2005. 